\title{
Correcting Data Shifts in Gel Files Created by Model 377 DNA Sequencers
}

BioTechniques 24:1002-1003 (June 1998)

\author{
K.M. O'Brien, M.A. Ironside, M.C. \\ Athanasiou, M.A. Basit, G.A. Evans and \\ H.R. Garner \\ University of Texas, Southwestern Medical \\ Center, Dallas, TX, USA
}

The Eugene McDermott Center for Human Growth and Development at the University of Texas, Southwestern Medical Center uses the Model 377 Sequencers (PE Applied Biosystems, Foster City, CA, USA) equipped with the XL upgrade (i.e., collection software Version 2.0 and analysis software Version 3.0). Approximately $1 \%$ of the gel files reviewed by one of these authors (M.C. Athanasiou) manifest a specific error whose nature is to horizontally shift all data present in the gel file after some scan number. As Figure 1A shows, this can happen more than once even in the same run. The two shifts shown in this gel file occur between scan numbers 3743 and 3744 and between scan numbers 4464 and 4465. The first of these shifts is a total of 8 channels to the left, while the second is 7 channels to the right. Note that, as far as we can determine, the tracking software used by PE Applied Biosystems products is not capable of properly tracking a gel file of this nature. As a result, the researcher must $(i)$ either manually re-track the gel file, taking the chance artificial chimeric errors of seemingly high quality but with incorrect base calls over large sections may be introduced or (ii) perform the entire experiment over again at a cost on order of \$230 for the gel mixture and sequencing kits alone each time this happens (1). If samples have to be prepared anew, this cost can be significantly higher.

Software has been developed that allows the experimenter to shift user-defined blocks of data horizontally over some chosen number of channels. The program that makes this possible, UnShift, was applied twice to the gel file shown in Figure 1A. The first application shifted all scans from number 3744 to the end of the data 8 channels to the right. Figure 1B shows the resulting gel file. The second application shifted all scans from number 4465 onwards 7 channels to the left. Figure $1 \mathrm{C}$ shows the final, now corrected, gel file. This gel file could then be used to extract the lanes much easier and, since the lanes now line up properly, with less of a chance of intro- ducing a chimerism.

UnShift works by manipulating the data storage architecture of PE Applied Biosystems gel files. It first reads the total number of scan lines from the gel file's header as it transfers this header over to the corrected gel file. It also writes an incorrect number to the part of the header that deals with the image size to cause Sequencing Analysis software (Version 3.0; PE Applied Biosystems) to automatically regenerate the gel image. This leads to an error message that reads 'The Gel file length is not the expected length', which is reported by the sequencing analysis software when the corrected gel file is first opened; however, the error message introduces no other defects into either the data or the image sections of the gel file. All scan lines before the one specified by the user are then transferred directly to the target file. Finally, the remainder of the data are shifted using a technique that allows the data to wrap around the edges of the gel and then be written to the corrected file.

UnShift is available for download as freeware at the Web site: http://gestec.swmed.edu/gestec2.htm along with a complete set of documentation. A copy of the source code is also provided should the user wish to review the method used in more detail or make any modifications. When correcting a data shift, the program's executable file should be placed in the same folder on the Macintosh ${ }^{\circledR}$ as the gel file and a duplicate of the gel file. For the program to function properly, the gel file's name cannot include any spaces. Also, successive applications of UnShift allow the user to correct two or more data shifts that may have occurred in the same gel file, as was demonstrated above. Note that this software makes no effort to smooth or otherwise improve the data quality. As a result, seams between shifted sections are often visible upon close inspection. These may interfere with base-calling in some cases; however, this seemed preferable to introducing any data alterations, which would be required to eliminate these seams. Also, depending on the type of computer used, it may be necessary to regenerate the gel image after application of UnShift. This can easily be done within Sequencing Analysis software Version 3.0 by selecting 'Regenerate Gel Images...' from the 'Gel' pull-down menu. Finally, UnShift will only operate on gel files created by a Model 377 Sequencer. A copy of the source code is available at the Web site mentioned above for those who might want to customize the program. 
UnShift was written with Metrowerks Code Warrior Version 2.0 on a UMax SUPERMAC PPC clone (UMax Technologies, Santa Clara, CA, USA), but also runs on a variety of other Macintosh Computers (Apple Computer, Cupertino, CA, USA).

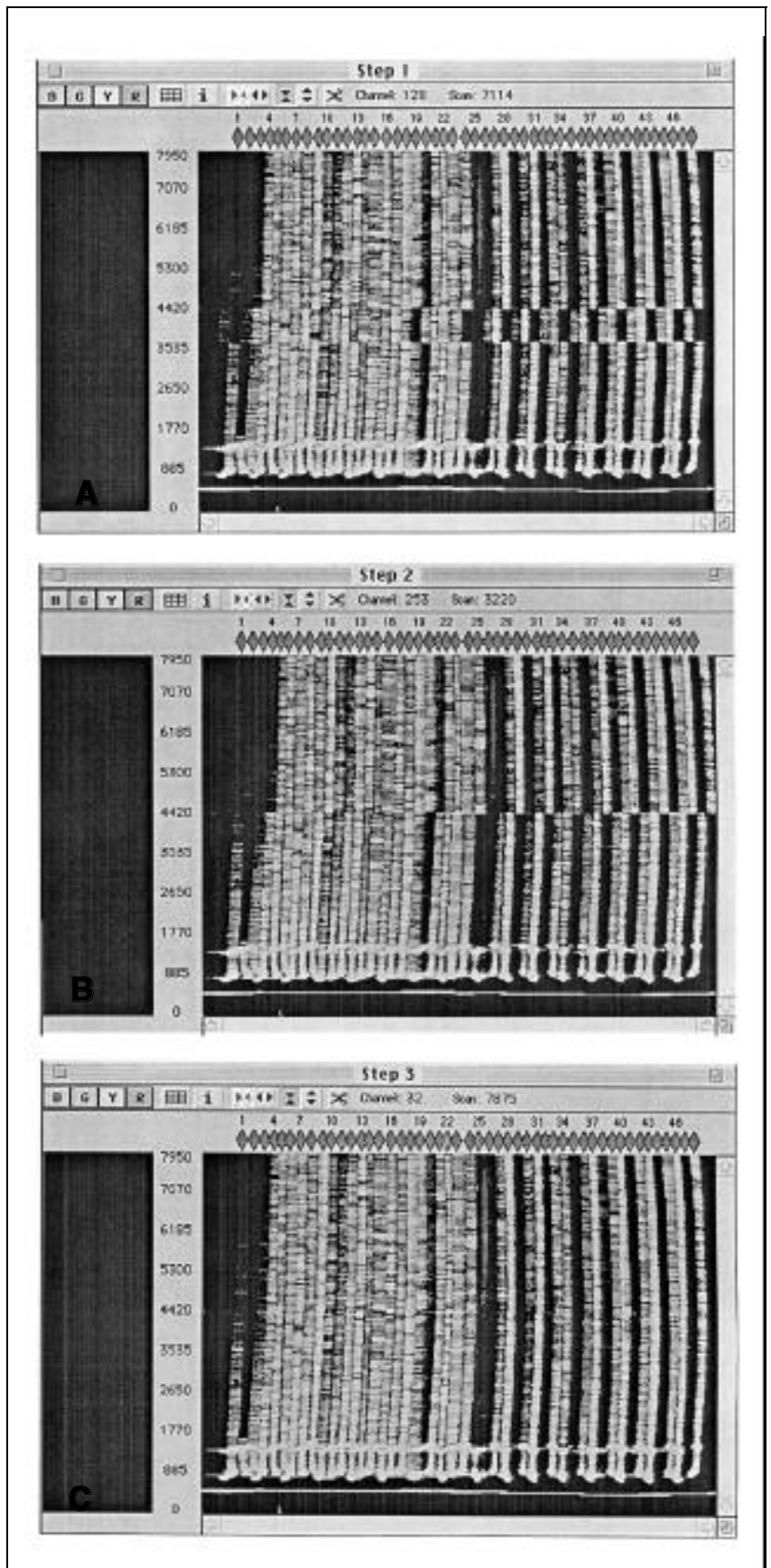

Figure 1. (A) Sample gel file showing two horizontal shifts. The first of these shifts occurs between scan lines 3743 and 3744. It is an 8-channel shift to the right. The second of these shifts occurs between scan lines 4464 and 4465. It is a 7-channel shift to the left. (B) Gel file after first application of UnShift. The first shift evident in Panel A has been completely removed. (C) Gel file after second application of UnShift. The second and final shift present in Panel A is now gone.

\section{ACKNOWLEDGMENTS}

This work was supported by grants from the NHGRI (No. HG00202-07) and Department of Energy (Nos. 95ER69608 and 95ER62055).

\section{REFERENCE}

1.O'Brien, K.M., J.W. Fondon III, G.A. Evans and H.R. Garner. 1997. Rescuing corrupted gel files from Model 377 and 373 DNA sequencers. BioTechniques 22:1162-1163.

Received 10 November 1997; accepted 30 December 1997.

Address correspondence to:

Dr. Kevin M. O'Brien

Eugene McDermott Center for Human Growth and Development

The University of Texas, Southwestern Medical Center

5323 Harry Hines Blvd.

Dallas, TX 75235-8591, USA

Internet: kevin@mcdermott.swmed.edu 